\title{
Relación morfofuncional del plano oclusal con el músculo masetero
}

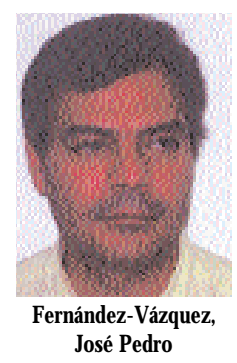

\section{Morphofunctional relationship between the occlusal plane and the masseter muscle}

\section{Fernández-Vázquez, J osé Pedro* Olay-García, Sonsoles** \\ González- Lafita, Pedro*** Conzález-Rico, María****}

*Profesor Titular Interino. Prótesis y Oclusión. Facultad de Medicina y Odontología. Universidad de Oviedo.

**Profesora Asociada. Integrada de Adultos. Facultad de Medicina y Odontología. Universidad de Oviedo

***Colaborador de Honor. Servicio de Prótesis y Oclusión. Facultad de Medicina y Odontología. Universidad de Oviedo.

****Medico especialista en Estomatología. Practica privada

\section{Correspondencia}

J osé Pedro Fernández Vázquez Universidad de Oviedo. Servicio de Prótesis y Oclusión

Escuela de Estomatología

Catedrático J osé Serrano s/n

33009 Oviedo

E-mail: jpfv@sci.cpd.uniovi.es
Resumen: Introducción (fundamento y objetivo): La relación morfofuncional entre el músculo masetero (MM) y el plano oclusal no ha sido investigada en profundidad. El presente trabajo tiene por objetivo fundamental demostrar que las fibras musculares del masetero muestran una posición perpendicular al plano oclusal. Método: Se ha procedido a la evaluación de las relaciones entre el fascículo superficial del MM y la posición de los incisivos, primer molar inferior, plano oclusal y plano de Francfort, a partir de las teleradiografías de 21 voluntarios sanos, a quienes se les había colocado un dispositivo de silicona intraoral para la identificación del MM. Resultados: El ángulo que forman las fibras del fascículo superficial del MM y el plano oclusal es muy variable situándose entre 70 y $99^{\circ}$ con un rango de $29^{\circ}$ y la media es de $80,7^{\circ}$. Con el plano de Francfort obtuvimos una media de $65,8^{\circ}$ con un rango de 31,5 . Conclusión: Las fibras del fascículo superficial del MM no son perpendiculares al plano oclusal.

Palabras clave: Músculo masetero, Oclusión, Plano oclusal, Plano de Francfort, Teleradiografia.

Abstract: Introduction and purpose: The morphofunctional relationship between the masseter muscle (MM) and the occlusal plane hasn 't been investigated in depth. The main goal of the present study is to prove that the fibres of the MM are located prependiculaly to the occlusal plane. Methods: We have evaluated the relationships between the superficial portion of the MM and the position of the incisers, first lower molar, occlusal plane and the Francfort plane, from tele-radiographs of 21 healthy voluntaries, on whom we had placed an intraoral silicone guide for identifying the MM. Results: The angle that the fibres of the superficial portion of MM formes with the occlusal plane is very variable, from 70 to $90^{\circ}$, with a range of $29 \circ$. The average is 80,70 . With the Francfort plane, we had obtained an average of $65,8^{\circ}$ with a range of $31,5^{\circ}$. Conclusions: The fibres of the superficial portion of the MM do not run perpendicular to the occlusal plane.

Key word: Masseter muscle, Occlusion, Occlusal plane, Francfort plane, Teleradiograph.

BIBUD [1138-123X (2003)8:5; agosto-septiembre 469-592]

Fernández-Vázquez JP, Olay-García S, González-Lafita P, González-Rico M. Relación morfofuncional del plano oclusal con el músculo masetero. RCOE 2003;8(5):513-520. 


\section{Introducción}

El masetero es un músculo rectangular que se origina en el arco cigomático y se extiende hacia abajo y atrás hasta la cara externa del borde inferior de la mandíbula ${ }^{1 * *, 2,3}$, su inserción se extiende desde la región del segundo molar hasta el mismo ángulo. Consta de dos fascículos: profundo y superficial, que es el de nuestro interés, aunque otros autores, como Wislow, describen hasta tres ${ }^{4}$. La porción superficial nace, por una lámina tendinosa gruesa, de los tres cuartos anteriores del borde inferior del arco cigomático. Esta inserción se extiende a la parte más próxima de la pirámide del maxilar superior, por delante del ángulo inferior del hueso malar. Los haces carnosos se dirigen oblicuamente hacia abajo, atrás y ligeramente hacia dentro y terminan en el ángulo, el borde inferior y la parte inferior de la cara externa de la rama ascendente de la mandibula ${ }^{3,4}$. La función principal del masetero es la elevación mandibular pero, al contrario que el músculo temporal, que tiene un importante papel como posicionador durante el cierre mandibular, es un músculo potente que proporciona una masticación eficiente. Su fascículo superficial también interviene en la protusión mandibular, contrayéndose simétricamente junto con el pterigoideo interno y el fascículo inferior del lateral, y en la lateralidad, donde el fascículo superficial del lado de no trabajo se contrae unilateral y accesoriamente ${ }^{2}$.

Desde un punto de vista protésico, el plano oclusal es un plano imaginario delimitado por los bordes incisales de los dientes anteriores mandibulares y la punta de las cúspides vestibu- lares de los premolares y molares inferiores ${ }^{1 *, 2}$, o como señala el glosario de términos prostodoncicos ${ }^{5}$, el plano promedio formado por las superficies incisales y oclusales de los dientes. Cefalométricamente, se le define como aquel que une los puntos de máxima intercuspidación de los primeros molares y de los incisivos.

Aunque en el estudio de la telerradiografía lo marcamos como una línea recta, la realidad es que vistos los arcos dentarios en norma lateral, los molares muestran un nivel en altura diferente del resto de los dientes de la arcada, lo que unido a la colocación de todos ellos con diversos grados de inclinación, confieren al plano oclusal una curvatura de concavidad superior; la llamada curva de Spee, curva de oclusión o curva antero-posterior ${ }^{2,5,6}$.

Si hacemos la observación de frente podemos ver que tampoco determinan un plano recto, sino que los dientes posteriores mandibulares presentan una ligera inclinación lingual y los maxilares, por el contrario, una inclinación vestibular. Si trazamos una línea imaginaria en la mandíbula que pase por las cúspides vestibulares y linguales de los dientes posteriores derechos e izquierdos se observa una curva de oclusión cóncava llamado curva de Wilson o curva medio-lateral; para la arcada lateral seria convexa. Esto, parece que es debido a que el movimiento de la mandíbula está determinado por las dos articulaciones temporomandibulares, que rara vez actúan con movimientos idénticos y simultáneos, por lo que una superficie oclusal curva permite una utilización máxima de los contactos dentarios durante la función $n^{1 * *}$. Sin embargo, otros autores como Ramfjord $^{2}$ opinan que la asociación de estos planos imaginarios con la función es muy remota.

Los estudios que sobre este tema se han hecho y han ido encaminados, en su mayoría, a encontrar un referente claro que permita reconstruir un plano oclusal funcional en pacientes desdentados. El más usado en clínica es el plano de Camper que une o pasa por el borde inferior del ala de la nariz y borde superior del trago de ambos lados ${ }^{6}$. Ogawa y cols ${ }^{7}$ estudiaron, en 1996, la posibilidad de obtener una información precisa de la orientación del plano oclusal midiendo su relación con las trayectorias de cierre de la mandíbula. Según sus hallazgos, estas trayectorias son prácticamente perpendiculares a dicho plano a pesar de las diferencias de inclinación en él observadas. La conclusión a la que llegó en su estudio, fue que la formación del plano oclusal esta condicionada por el crecimiento y los cambios que se producen en los músculos masticatorios. En esta misma línea, Ingerval y Minder ${ }^{8}$, concluyeron diciendo, que en este campo de conocimiento, quedan todavía muchas cosas por investigar.

Otros trabajos ${ }^{9,10}$ estudian la influencia de los músculos de la masticación en distintos aspectos de la morfología craneofacial. Así Raadsheer y cols $^{11}$, han publicado un artículo relacionando la fuerza masticatoria con dicha morfología. Encontraron que existía correlación con el tamaño facial, pero la correlación era estadísticamente negativa con la inclinación mandibular y del plano oclusal, lo que contradice la conclusión de Ogawa7. En todo caso, en estos estudios se investiga sobre los efectos de la función muscular, pero no se determina la posición/orientación de las fibras muscula- 
res para hacer el análisis de los datos. El único autor que hemos encontrado que hace una localización del fascículo superficial del masetero es Kasai y cols $^{12,13}$, que utilizaron cadáveres para su estudio. Sus trabajos van orientados a ver las diferencias de tamaño y posición de dicho músculo en pacientes dentados y desdentados y su relación con la morfología craneofacial.

En este contexto y puesto que la masticación en su expresión más reduccionista, consiste en un determinado contacto oclusal entre ambas arcadas, proporcionado por el plano oclusal moviéndose por la acción y efecto de los músculos elevadores mandibulares y en especial del masetero que proporciona la mayor cantidad de fuerza masticatoria, seria por tanto necesario, desde un punto de vista biomecánico, para una mayor eficacia masticatoria con menor fuerza y fatiga por parte de ambos maseteros disponer de un ángulo de aplicación respecto al plano oclusal lo suficientemente agudo para mover la mandíbula y con ella el plano oclusal con el mínimo esfuerzo muscular. Con esta base la hipótesis de partida es: las fibras musculares del músculo masetero tienen una dirección perpendicular respecto al plano oclusal. Esta hipótesis la podemos reducir a la consecución de los siguientes objetivos: 1. Establecer la orientación de las fibras del fascículo superficial del músculo masetero respecto al plano oclusal. 2. Determinar la orientación del plano de Francfort, del plano oclusal y algunas estructuras dentarias. 3 . Relacionar la inclinación de las fibras del masetero con el resto de los parámetros citados, analizando su correlación morfofuncional.

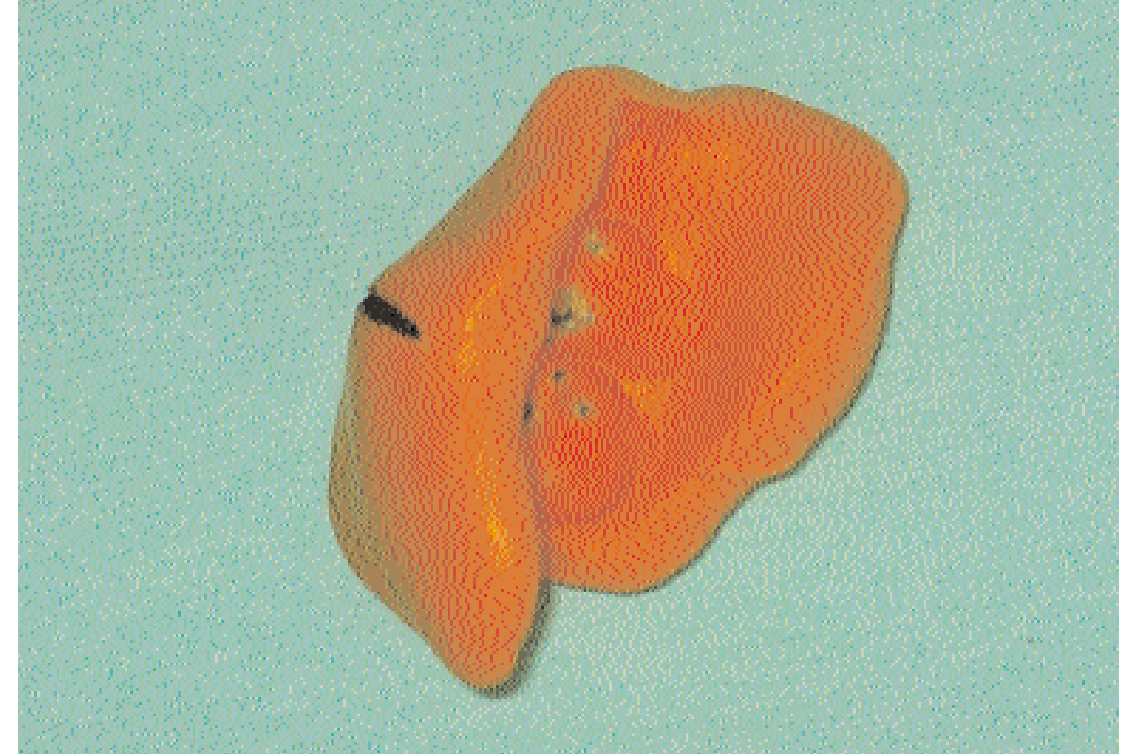

Figura 1. Aspecto de la llave de silicona

\section{Material y método}

\section{Sujetos}

Los sujetos fueron alumnos voluntarios de la Escuela de Estomatología de Oviedo. A toda la muestra se le efectuó una exploración para descartar la presencia de cualquiera de los siguientes criterios de exclusión: signos y/o síntomas de patología muscular y articular de articulación temporomandibular (ATM), ausencia de más de tres dientes, alteración del plano oclusal y maloclusión de cualquier tipo. También se excluyeron a aquellos en los que, tras la realización del molde de silicona no se pudo señalar el borde anterior del músculo masetero debido a su poco grosor, lo que daba lugar a una huella muy débil, o a la interferencia del músculo buccinador. La ausencia de patología muscular y articular se efectúo por anamnesis y una exploración completa del aparato estomatognático por un único y experto explorador ${ }^{14^{*}-16}$.

El tamaño de la muestra quedó constituido por 21 casos válidos de los cuales 9 fueron hombres (42,9\%) y 12 mujeres $(51,7 \%)$. Las edades estaban comprendidas entre 25 y 41 años, con una media de 30,29, siendo el valor más frecuente 28 años.

\section{Materiales y recogida de datos}

Para poder situar correctamente el músculo masetero en las personas de la muestra, se ideó un dispositivo consistente en una llave de silicona pesada sobre la que se colocaba un contraste metálico que posteriormente al realizar una telerradiografía, nos permitía medir los distintos ángulos (fig. 1).

Para la realización de este dispositivo, se introdujo la silicona en el vestíbulo derecho de forma que una pequeña cantidad quede sobre la superficie oclusal, lo que nos permitirá más tarde colocarla en su posición exacta y que no se mueva durante la realización de la telerradiografía. El voluntario realiza una contracción continuada del masetero hasta que la 
silicona fragua quedando marcada la cuerda que forma el borde anterior del músculo. En esa huella se hace una hendidura y se introduce el contraste metálico (figs. 2 y 3 ).

Así colocada la llave y el contraste metálico que nos marca la dirección de las fibras del masetero, se procedió a la realización de una telerradiografía a cada paciente, cuya fiabilidad fue validada por Kasai ${ }^{12,13}$ en cadáveres. Además presenta una serie de características técnicas que la hacen muy adecuadas para nuestro estudio ${ }^{17}: 1$. Da una imagen nítida y de tamaño muy cercano al real ya que al aumentar la distancia foco-objeto (aproximadamente 1,5 metros), respecto a la radiografía convencional de cráneo, se reducen al mínimo la diferencia visible en la práctica entre dos puntos situados próximo y lejano a la película. Esto permite realizar medidas de distancias y de ángulos. 2. La cabeza va fijada en un soporte lo que garantiza la colocación del cráneo lo más exacta posible y repetible de los distintos planos de referencia en todas las exploraciones. 3. Permite ver partes blandas por el uso de filtros o pantallas diferenciales especiales.

Una vez obtenidas las telerradiografias, se observa nítidamente la línea radiopaca que marca el borde anterior del masetero y que tomamos como referencia para medir los diferentes ángulos estudiados. Para el análisis cefalométrico se marcó el plano oclusal, el plano de Francfort, los ejes de los incisivos centrales superior e inferior, así como la perpendicular a la raíz distal del primer molar inferior con el fin de establecer su relación angular con el masetero y los planos estudiados (fig. 4)

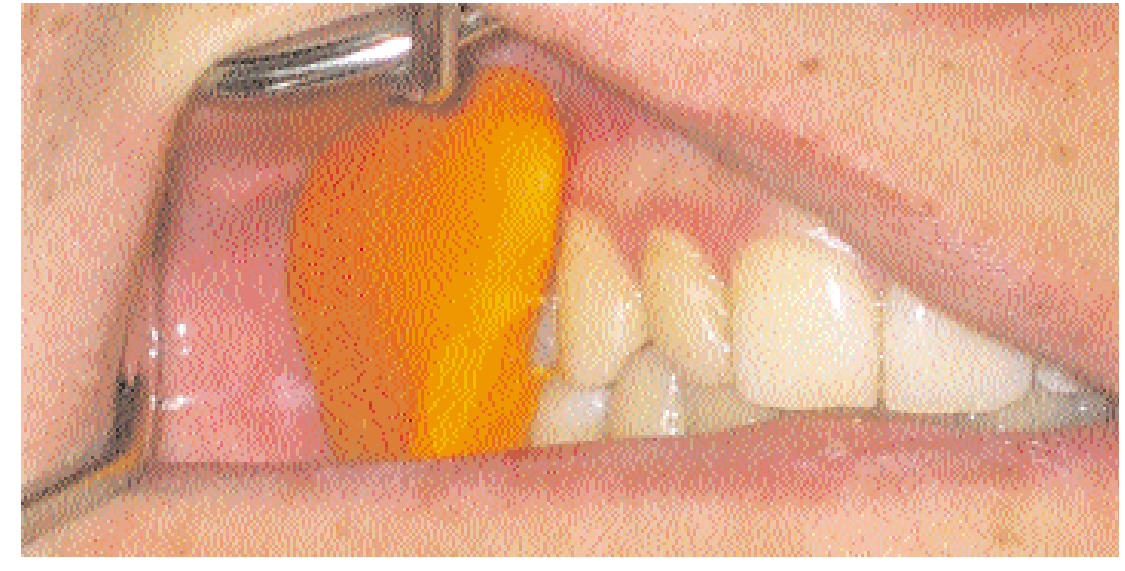

Figura 2. Llave de silicona posicionada en la boca.

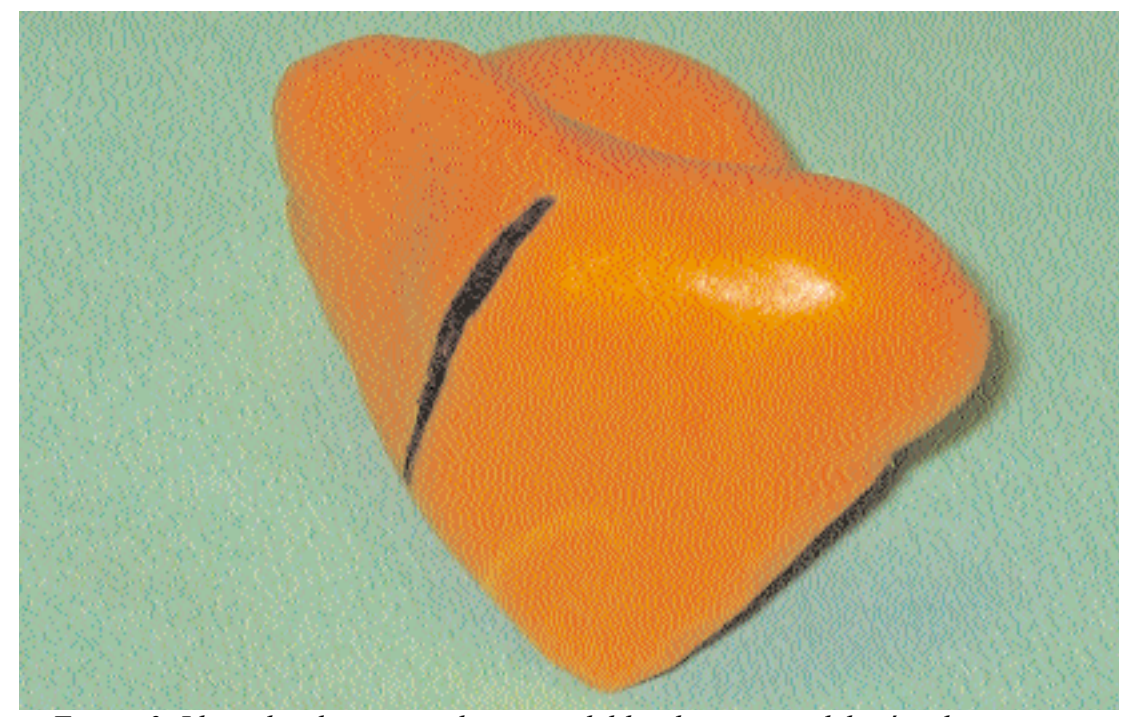

Figura 3. Llave de silicona con la marca del borde anterior del músculo masetero

\section{Análisis de los datos}

Una vez obtenidos los datos se procedió a su agrupación y procesamiento estadístico utilizando el programa SPSS/ + versión 10.0. El análisis realizado se desglosó en dos grandes grupos: descriptivo, se obtuvo una descripción de las variables estudiadas mediante la frecuencia y las medidas de tendencia central y de dispersión, e inferencial de contraste de hipótesis mediante una prueba de conformi- dad de chi cuadrado, que compara una proporción observada con una teórica.

\section{Resultados}

Descriptivos. En la tabla 1, se detallan las relaciones angulares de los incisivos centrales con respecto al plano oclusal así como el ángulo interincisal, ya que son unos factores impor- 


\begin{tabular}{|c|c|c|}
\hline \multicolumn{3}{|c|}{$\begin{array}{c}\text { Tabla I: Relaciones angulares } \\
\text { entre los incisivos y el plano } \\
\text { oclusal }\end{array}$} \\
\hline \multicolumn{3}{|c|}{$\begin{array}{cc}\text { Ángulo } & \text { Ángulo } \\
\text { plano } & \text { plano } \\
\text { oclusal- } & \text { oclusal- Ángulo } \\
\text { incisivo } & \text { incisivo interin- } \\
\text { inferior } & \text { superior cisivo } \\
\end{array}$} \\
\hline 21 & 21 & 21 \\
\hline Media & 62 & 133 \\
\hline Mediana 114 & 65,5 & 129 \\
\hline Moda $\quad 112,5$ & 52,5 & 123 \\
\hline Desv. Tip. $\quad 6,12$ & 5,88 & 12,57 \\
\hline Mínimo $\quad 97,5$ & 52,5 & 112 \\
\hline Máximo 125,5 & 74,5 & 159 \\
\hline
\end{tabular}

tantes para la estabilidad del aparato estomatognático.

El incisivo inferior forma un ángulo obtuso respecto al plano oclusal con valores que oscilan entre 97,5 y los $125^{\circ}$, con una media de $112,9^{\circ}$ y una moda de $112,5^{\circ}$. Por el contrario el incisivo superior forma un ángulo agudo, con un valor medio de $62,1^{\circ}$. La variabilidad es menor en la angulación del incisivo superior con un rango de $22^{\circ}$ frente a $28^{\circ}$ en el caso del incisivo inferior. En cuanto al ángulo interincisival se refiere hemos obtenido una media de $133^{\circ}$ con una gran variabilidad (rango de 47ㅇ)

En la tabla 2 se recogen los valores de los ángulos de los incisivos en relación con el fascículo superficial del masetero.

Para que el cierre mandibular sea más eficaz el ángulo del incisivo inferior respecto al masetero debería ser lo más pequeño posible (lo que indicaría mayor paralelismo entre ambas estructuras). Los datos que hemos obtenido reflejan una distribución casi simétrica con moda y mediana de $13^{\circ}$
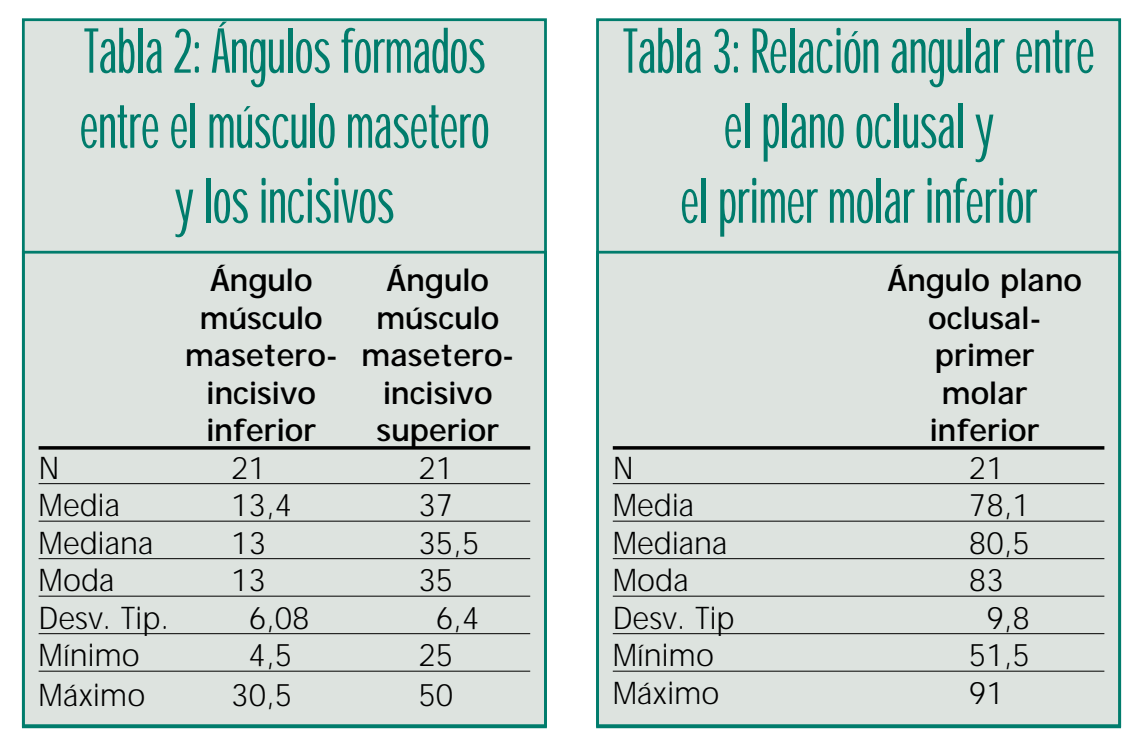

y una media de $13,4^{\circ}$. La variabilidad es grande con valores comprendidos entre 4,5 y $30,5^{\circ}$

La posición del incisivo inferior es adecuada respecto al plano oclusal y tiene un buen paralelismo con relación al masetero. Esto nos indica un correcto funcionamiento de la vía anterior (importante para mantener una buena función del sistema masticatorio y por tanto de la salud del aparato estomatognático). Pero son los sectores posteriores los que llevan el mayor peso de la función masticatoria por lo que hemos analizado la posición de los primeros molares, centrándonos en el inferior, ya que la posición del superior es muy estable.

Siguiendo la misma dinámica que con los incisivos, en la tabla 3, se detallan los datos correspondientes a la relación del primer molar inferior con el plano oclusal. El rango de valores que observamos fue muy amplio (entre 51,5 y $91^{\circ}$ ). Más de la mitad de los valores se sitúan entre los 80 y $91^{\circ}$, pero debido a la influencia que tienen los valores extremos en la media, ésta

\begin{tabular}{|c|c|}
\hline \multicolumn{2}{|c|}{$\begin{array}{c}\text { Tabla 4: Relación angular entr } \\
\text { el mesetero y } \\
\text { el primer molar inferior }\end{array}$} \\
\hline & $\begin{array}{l}\text { Ángulo } \\
\text { masetero- } \\
\text { primer } \\
\text { molar } \\
\text { inferior } \\
\end{array}$ \\
\hline$\overline{\mathrm{N}}$ & 21 \\
\hline Media & 7,8 \\
\hline Mediana & 3,5 \\
\hline Moda & 3 \\
\hline Desv. Tip & 9,9 \\
\hline Mínimo & 0,5 \\
\hline Máximo & 45 \\
\hline
\end{tabular}

baja hasta $78,1^{\circ}$. La mediana y la moda se sitúan por encima de $80^{\circ}$ (80,5 y $83^{\circ}$ respectivamente).

Por lo que respecta al ángulo entre el molar inferior y las fibras del fascículo superficial del masetero hemos visto una situación similar a la anterior con una gran variabilidad en los datos que pueden hacer engañosa la media obtenida $\left(7,8^{\circ}\right)$ (tabla 4). En este caso, 
como ocurría en el incisivo inferior, lo ideal son ángulos lo más pequeños posibles que indiquen paralelismo entre las dos estructuras y por lo tanto una mayor eficacia en la masticación. La mediana y la moda son prácticamente iguales con valores de 3,5 y $3^{\circ}$ respectivamente.

Finalmente, en cuanto a las relaciones entre los distintos planos que hemos delimitado en la telerradiografía (tabla 5) observamos que el ángulo que forman las fibras del fascículo superficial del masetero y el plano oclusal es muy variable situándose entre 70 y $99^{\circ}$ con un rango de $29^{\circ}$. La media fue de $80,7^{\circ}$ y la muestra tiene una distribución bimodal, siendo éstas 80 y 84,5.

Estas mismas fibras musculares las hemos relacionado con el plano de Francfort y los resultados obtenidos fueron los siguientes: media de $65,8^{\circ}$ con un rango de 31,5 y al igual que el anterior sigue una distribución bimodal $\left(65\right.$ y $\left.69^{\circ}\right)$.

Respecto a la relación entre el plano oclusal y el de Francfort podemos decir que se ajusta a lo que ya era conocido ya que su valor medio fue de $14,8^{\circ}$. Aquí también la variación fue amplia, encontrándose los valores obtenidos entre $7^{\circ}$ y $27,5^{\circ}$, con una variación de $20,5^{\circ}$. En este caso las modas son múltiples sin que ningún valor se repita más que los demás.

Inferenciales. A la hora de comparar las variables, el ángulo formado entre el masetero (fascículo superficial) y el plano oclusal, mediante una prueba de conformidad chi cuadrado $\left(\chi^{2}\right)$ obtuvimos un valor $\chi^{2}=5,7619$ para un grado de libertad $(p=0,0164)$. Por ello, para un intervalo de confianza del $95 \%$ podemos establecer que el

\begin{tabular}{|c|c|c|c|}
\hline & $\begin{array}{c}\text { Ángulo plano } \\
\text { oclusal- } \\
\text { masetero }\end{array}$ & $\begin{array}{l}\text { Ángulo plano } \\
\text { oclusal- plano } \\
\text { de Francfort }\end{array}$ & $\begin{array}{c}\text { Ángulo } \\
\text { masetero-plano } \\
\text { de Francfort }\end{array}$ \\
\hline $\mathrm{N}$ & 21 & 21 & 21 \\
\hline Media & 80,7 & 14,8 & 65,8 \\
\hline Mediana & 80 & 14 & 65 \\
\hline Moda & 80 & 7 & 65 \\
\hline Desv. Tip & 7,09 & 5,49 & 7,96 \\
\hline Mínimo & 70 & 7 & 50,5 \\
\hline Máximo & 99 & 27,5 & 81 \\
\hline Chi-cuadrado & 5,7619 & & \\
\hline gl & 1 & & \\
\hline $\mathbf{p}$ & 0,0164 & & \\
\hline
\end{tabular}

plano oclusal se sitúa, respecto al fascículo superficial del masetero, con un ángulo inferior a 85으 o superior 95‥ Por lo tanto, en nuestro estudio, el plano oclusal no es exactamente perpendicular al músculo masetero.

\section{Discusión}

Hasta ahora la única forma que ha habido para determinar la posición del músculo masetero en un determinado paciente ha sido el uso de técnicas de imagen como la resonancia nuclear magnética (RNM) o la ecografía ${ }^{16,18,19}$, o bien como Kasail ${ }^{12,13}$ que en sus estudios en cadáveres empleó para definir el masetero bario líquido, pero esto no era viable en personas vivas. Para el presente estudio, hemos intentado encontrar un método más simple y económico de hacer esta fijación. Para tal fin, se ideó un novedoso dispositivo consistente en el molde de silicona pesada en el que se coloca un contraste metálico. La utilidad que esto pueda tener en el ámbito clínico diario está por determinar y se necesitarán otros trabajos para poder establecer la posibilidad real de su aplicación.

La telerradiografía como método de estudio de la morfología de las estructuras óseas y dentarias y su interrelación no es nada nuevo. Desde un punto de vista clínico, su uso más extendido está en el campo de la ortodoncia para el diagnóstico y la planificación del tratamiento, ya que nos predice las tendencias del crecimiento ${ }^{9}$. En investigación se ha utilizado para analizar las diferencias anatómicas entre dentados y desdentados y ayudar a la reconstrucción de un plano oclusal funcional ${ }^{11,19,20}$.

Algunos datos que hemos obtenidos están sobradamente contrastados en varias fuentes bibliográficas. Un ejemplo de esto es el ángulo formado por el plano oclusal con el de Francfort; el valor comúnmente aceptado ${ }^{6}$ es $14^{\circ}$ y nosotros hemos obtenido un resultado similar de $14,8^{\circ}$.

Otra muestra es el ángulo interinci- 
sivo del que Dawson ${ }^{14}$, da tanto su definición como su importancia en la estabilidad de todo el aparato estomatognático. El valor medio que obtuvimos (133, con una variabilidad muy grande) se encuentra muy cercano al obtenido por él y otros autores $^{14,20}$. Este autor considera que todo este abanico de valores se puede aceptar como normal siempre que proporcione una buena disclusión y exista una buena estabilidad en los sectores posteriores. En nuestro caso todos los voluntarios presentaban una oclusión de clase I de Angle y no tenían signos ni síntomas de disfunción, lo que apoya su opinión.

Con respecto a las relaciones del primer molar inferior con el plano oclusal y el masetero no hemos encontrado nada publicado por lo que no podemos hacer comparaciones. Queremos dejar constancia de los valores hallados por si fueran de utilidad para futuros estudios.

En lo que a las relaciones del plano oclusal y el masetero se refiere, las referencias bibliográficas son escasas. El artículo que más se acerca a nuestro trabajo es el de Raadsheer ${ }^{11}$. Estudió 121 adultos realizándoles una ecografía de los músculos implicados en la masticación (masetero, temporal y digástrico) midiendo su grosor. También hizo telerradiografías para medir las distintas estructuras craneofaciales. En sus resultados podemos ver que sí existe relación entre la fuerza masticatoria y el grosor muscular, pero no con la orientación de la mandíbula y el plano oclusal; así valora los efectos de la función muscular pero no hace una determinación topográfica del músculo masetero.

Con los resultados que hemos obtenido en nuestro análisis de los 21 casos presentados, rechazamos la hipótesis nula de que las fibras del fascículo superficial del masetero son perpendiculares al plano oclusal y aceptamos la contraria, aunque debemos admitir que con la inclinación media obtenida de 80 grados, no podemos considerar que se puede formar un ángulo de aplicación de la acción del masetero excesivamente favorable para un mejor trabajo masticatorio.

Por otro lado para que el cierre mandibular sea más eficaz, el ángulo del incisivo inferior respecto al masetero debería ser lo más pequeño posible (lo que indicaría un mayor paralelismo entre ambas estructuras). Los datos que hemos obtenido reflejan una distribución cuasi simétrica con moda y mediana de $13^{\circ}$ y una media de $13,4^{\circ}$, y aunque la variabilidad fue grande, con valores comprendidos entre 4,5 y $30,5^{\circ}$, si podemos admitir que es adecuado.

La inclinación del incisivo inferior respecto al plano oclusal fue correcta y está de acuerdo con otros autores9 y además tiene un buen paralelismo con relación al masetero. Todo esto indica indirectamente un correcto funcionamiento de la guía anterior en la muestra, tan importante para mantener una buena función del sistema masticatorio y por tanto de la salud del aparato estomatognático.

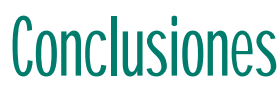

1. Las fibras del fascículo superficial del masetero no son exactamente perpendiculares al plano oclusal.

2. Las fibras del masetero forman un ángulo menor de $90^{\circ}$ con el plano de Francfort.

3. La angulación entre el plano oclusal y el de Francfort, observada en nuestro estudio, no se diferencian significativamente de lo referido en la literatura.

\section{Bibliografía recomendada}

Para profundizar en la lectura de este tema, el/los autor/es considera/an interesantes los artículos que aparecen señalados del siguiente modo: * de interés $* *$ de especial interés.

\footnotetext{
1**. Okenson JP. Oclusión y afecciones temporomandibulares. $3^{\mathrm{a}}$ ed. Madrid: Mosby/Doyma, 1992.

Libro de enorme interés y actualidad en relación a los problemas disfuncionales de las articulaciones temporomandibulares

2. Ramfjord S, Major M. Oclusión. Méjico D. F.:
}

Mcgraw-Hill. Interamericana, 1996

3. Feneis H. Nomenclatura anatómica ilustrada. Barcelona: Salvat, 1974.

4. Rouviêre H. Anatomía humana descriptiva y topográfica. $2^{\mathrm{a}}$ ed. Madrid: Bailly-Bailliere, 1950. 5. Van Blarcom CW. The glosary of prosthodontic terms $\mathbf{7}^{\mathbf{0}}$ Ed. J. Prosthet Dent 1999;81:39-110.
6. Saizar P. Prostodoncia total. Buenos Aires: Mundi, 1972.

7. Ogawa T, Koyano K, Suetsugu T. The relationship between inclination of the occlusal plane and jaw closing path. $J$ Prosthet Dent. 1996;76:576-80.

8. Ingervall $\mathrm{B}$, Minder $\mathrm{C}$. Correlation between 
maximun bite force and facial morphology in children. Angle Orthod 1997;67:415-22.

9. Canut Brusola JA. Ortodoncia Clínica. Barcelona: Salvat, 1991.

10. Kiliaridis S. Masticatory muscle influence on craniofacial growth. Acta Odontol Scand 1995;53:196-202.

11. Raadsheer MC, van Eijden TM, van Ginkel FC, Prahl-Anderson B. Contribution of the jaw muscle size and craniofacial morphology to human bite force magnitude. J Dent Res 1999; 78:31-42

12. Kasai K, Richards LC, Kanazawa E, Iwasawa T. Cephalometric analysis of masseter muscle and dentoskeletal morphology in dentate and edentulous humans. J Nihon Sch Dent 1997;39:78-85.
13. Kasai K, Richard LC, Kanazawa E, Ozaki T, Iwasawa $\mathrm{T}$. Relationship between attachment of superficial masseter muscle and craniofacial morphology in dentate and edentulous humans. J Dent Res 1994;73:1142-9.

14*. Dawson PE. Evaluación, diagnóstico y tratamiento de los problemas oclusales. Barcelona: Salvat,1991.

Libro de interés que aproxima al diagnóstico de los problemas oclusales y a los distintos abordajes que se pueden desarrollar para planificar los tratamientos de los mismos.

15. Hansson T, Honee W, Hesse J, Jiménez V. Disfunción cráneo-mandibular. Madrid: Editorial Praxis, 1988.

16. Gleb H, Bernstein I. Clinical evaluation of two hundred patients with temporomandibular joint syndrome. J Prosthet Dent 1983:49:234-40.

17. Pasler FA. Radiología odontológica. Barcelona: Salvat, 1988

18. Minowa K. Comparasion of magnetic resonance imaging and gross findings regarding masseter muscle aponeuroses in cadavers. Oral Surg Oral Pathol Oral Radiol Endod 1998;86:275-9.

19. Capaccioli L. The correlation between the echographic aspect of the perioral and masticatory muscle and dento-skeletal characteristics. Radiol Med 1998;95:567-72.

20. D'Souza NL, Bhargava K. A cephalometryc study comparing the occlusal plane in dentulous and edentulous subjects in relation to the maxillomandibular space. J Prosthet Dent 1996; 75:177-82.

RCOE, 2003, Vol 8, №5, 513-520 